\title{
FORMALLY INTEGRABLE COMPLEX STRUCTURES ON HIGHER DIMENSIONAL KNOT SPACES
}

\author{
DOMENICO FIORENZA AND HÔNG VÂN LÊ
}

\begin{abstract}
Let $S$ be a compact oriented finite dimensional manifold and $M$ a finite dimensional Riemannian manifold, let $\operatorname{Imm}_{f}(S, M)$ the space of all free immersions $\varphi: S \rightarrow M$ and let $B_{i, f}^{+}(S, M)$ the quotient space $\operatorname{Imm}_{f}(S, M) / \operatorname{Diff}^{+}(S)$, where $\operatorname{Diff}^{+}(S)$ denotes the group of orientation preserving diffeomorphisms of $S$. In this paper we prove that if $M$ admits a parallel $r$-fold vector cross product $\chi \in \Omega^{r}(M, T M)$ and $\operatorname{dim} S=r-1$ then $B_{i, f}^{+}(S, M)$ is a formally Kähler manifold. This generalizes Brylinski's, LeBrun's and Verbitsky's results for the case that $S$ is a codimension 2 submanifold in $M$, and $S=S^{1}$ or $M$ is a torsion-free $G_{2}$-manifold respectively.
\end{abstract}

\section{INTRODUCTION}

Let $(M, g)$ be a finite dimensional Riemannian manifold with a $r$-fold vector cross product (VCP for short) $\chi \in \Omega^{r}(M, T M)$. By definition, this means that $\chi$ satisfies the following properties

$$
\begin{gathered}
\left\langle\chi\left(v_{1}, \cdots, v_{r}\right), v_{i}\right\rangle=0 \text { for } 1 \leqslant i \leqslant r, \\
\left\langle\chi\left(v_{1}, \cdots, v_{r}\right), \chi\left(v_{1}, \cdots, v_{r}\right)\right\rangle=\left\|v_{1} \wedge \cdots \wedge v_{r}\right\|^{2}
\end{gathered}
$$

where $\langle\cdot, \cdot\rangle$ denotes the inner product defined by $g$ and $\|\cdot\|$ is the induced metric on $\Lambda^{r} T M$. For a VCP $\chi \in \Omega^{r}(M, T M)$ we associate the VCP-form $\varphi_{\chi} \in \Omega^{r+1}(M)$ as follows [LL2007, p. 143]

$$
\varphi_{\chi}\left(v_{1}, \cdots, v_{r+1}\right)=\left\langle\chi\left(v_{1}, \cdots, v_{r}\right), v_{r+1}\right\rangle .
$$

The notion of a VCP structure on a manifold was introduced by Gray BG1967, Gray1969]. In [BG1967] Brown and Gray classified linear VCPs on an $m$-dimensional real vector space $V^{m}$ with positive definite inner product $g$. Their results can be summarized as follows:

(1) A 1-fold VCP on $V^{m}$ exists iff $m=2 n$. It is defined uniquely by its associated VCP-form which is a Kähler form on $V^{2 n} \cong V^{n} \otimes_{\mathbb{R}} \mathbb{C}$.

(2) A $(m-1)$-fold VCP on $V^{m}$ is defined uniquely by its associated VCPform which is the volume form on $\left(V^{m}, g\right)$.

Date: October 20, 2020.

2010 Mathematics Subject Classification. Primary:58D10, Secondary:53D15, 46T10 .

Key words and phrases. vector cross product structure, loop space, weak $L_{2}$-metric, formally integrable complex structure.

Research of HVL was supported by GAČR-project 18-00496S and RVO: 67985840. 
(3) A 2-fold VCP exists on $V^{m}$ iff $m=7$ and it is defined uniquely by its associated VCP-form, which is the associative 3 -form on $\mathbb{R}^{7}$, whose stabilizer is the exceptional Lie group $G_{2}$.

(4) A 3-fold VCP exists on $V^{m}$ iff $m=8$ and it is defined uniquely by its associated VCP-form, which is the Cayley 4-form on $\mathbb{R}^{8}$, whose stabilizer is the Lie group $\operatorname{Spin}(7)$.

It follows from Brown-Gray's classification that a Riemannian manifold $\left(M^{m}, g\right)$ admits a $r$-fold VCP iff either $(1) r=1, m=2 n$ and $(M, g)$ is an almost Hermitian manifold and the associated VCP-form is the fundamental 2-form of the almost Hermitian manifold $(M, g),(2) r=m-1$ and $\left(M^{m}, g\right)$ is an orientable Riemannian manifold, (3) $r=2, m=7$ and $\left(M^{7}, g\right)$ admits an associative 3 -form $\varphi^{3}$, (4) $r=3, m=8$ and $\left(M^{8}, g\right)$ admits a Cayley 4form. Hence, a Riemannian manifold $(M, g)$ can be endowed with a parallel $r$-fold VCP iff either $\left(M^{m}, g\right)$ is an orientable Riemannian manifold and $r=m-1$; or $m=2 n,\left(M^{2 n}, g\right)$ is a Kähler manifold and $r=1$; or $m=7$ and $\left(M^{7}, g\right)$ is a torsion-free $G_{2}$-manifold and $r=2$; or $m=8$ and $\left(M^{8}, g\right)$ is a torsion-free $\operatorname{Spin}(7)$-manifold and $r=3$. Once more, this result singles out Kähler manifolds, torsion-free $G_{2}$-and $\operatorname{Spin}(7)$-manifolds as important classes of Riemannian manifolds with special holonomy Joyce2000. Not unrelatedly, these classes play a prominent role in calibrated geometry, string theory and $M$-theory Joyce2007.

In LL2007, motivated by Brylinsky's results on the loop spaces of Riemannian 3-manifolds Brylinsky2008, Lee and Leung initiated the study of moduli spaces $B_{e}^{+}(S, M)$ of unparametrized embedded oriented submanifolds in a finite dimensional Riemannian manifold $\left(M^{m}, g\right)$, diffeomorphic to a closed oriented manifold $S$, assuming that $\left(M^{m}, g\right)$ admits an $r$-fold VCP $\chi$ and $\operatorname{dim} S=r-1$. In particular, they proved that if the associated VCP-form $\varphi_{\chi}$ is closed then $\varphi_{\chi}$ induces a weak symplectic form on $B_{e}^{+}(S, M)$, which is compatible with the weak $L_{2}$-metric on $B_{e}^{+}(S, M)$, thus defining an almost Kähler structure. Their result partially extends Brylinsky's theorem for $B_{e}^{+}\left(S^{1}, M^{3}\right)$ and LeBrun's extension to the case $B_{e}^{+}(S, M)$ when $\operatorname{codim} S=2$ LeBrun1993, stating that if $\operatorname{codim} S=2$ then the induced weak symplectic form on $B_{e}^{+}(S, M)$ is formally Kähler, i.e., it is such that the associated almost complex structure has vanishing Nijenhuis tensor. Few years after Lee-Leung's work, Verbitsky proved that also the associated almost complex structure $J$ on $B_{e}^{+}\left(S^{1}, M^{7}\right)$ has vanishing Nijenhuis tensor if the associated VCP-form $\varphi^{3}$ is parallel and therefore $M^{7}$ is a torsion-free $G_{2}$-manifold Verbitsky2010. Almost at the same time, Henrich gave a new proof of Brylinsky's result by showing that $J$ is parallel with respect to the Levi-Civita connection of the $L_{2}$-metric.

Remark 1.1. The formal integrability of the complex structure $J$ on $B_{i, f}^{+}(S, M)$ does not imply its strong integrability, since the Newlander-Nirenberg theorem does not hold for infinite dimensional manifolds Lempert1993. Namely, 
in the infinite dimensional case if the the Nijenhuis tensor $N_{J}$ of a complex structure $J$ vanishes, then one still has plenty of local holomorphic functions, but this does not suffice to construct charts with values in an infinite-dimensional Banach or Fréchet space. To overcome this difficulty, in Lempert1993 Lempert introduced the intermediate notion of weak integrability of an almost complex structure and proved that the almost complex structure on the loop space $B_{i, f}^{+}\left(S^{1}, M^{3}\right)$ is weakly integrable. Since weak integrability implies formal integrability, Lempert's result provides in particular a refinement of Brylinsky's proof of the formal integrability of the almost complex structure $J$ on the space $B_{i, f}^{+}\left(S^{1}, M^{3}\right)$.

Remark 1.2. Lempert's proof of the weak integrability of the almost complex structure $J$ on $B_{i, f}^{+}\left(S^{1}, M^{3}\right)$ uses the twistor construction of a CR 5manifold over a 3-manifold $M^{3}$ proposed by LeBrun in [LeBrun1984. This twistor construction has been generalized further by LeBrun (resp. Verbitsky) for their proof of formal integrability of the almost complex structure $J$ on $B_{i, f}^{+}(S, M)$ in the case that $\operatorname{codim} S=2(\operatorname{resp} . \operatorname{dim} S=1 \& \operatorname{dim} M=7)$. Brylinski's original proof of the formal integrability of the almost complex structure $J$ on $B_{i, f}^{+}\left(S^{1}, M^{3}\right)$ uses instead an ingenious computation of the Nijenhuis tensor of $J$.

In this paper we show how Henrich's result admits a natural generalization, allowing us to extend Brylinski's and Verbitsky's results to all cases of Riemannian manifolds $(M, g)$ with parallel VCPs. In particular, this provides a new proof of Verbitsky's result as well as a new proof of LeBrun's result. Also in the case of $B_{e}^{+}\left(S^{1}, M^{3}\right)$ our proof simplifies Henrich's original argument, as we show that consideration on the parallelism of the almost complex structure $J$ with respect to a torsion-free affine connection $\nabla^{\perp}$ alone already allows us to conclude that $J$ is formally integrable. One can then derive from this that $J$ is actually also parallel with respect to the Levi-Civita connection on $B_{e}^{+}\left(S^{1}, M^{3}\right)$ and more generally, as we show, with respect to any torsion-free connection of $B_{e}^{+}(S, M)$ under consideration.

We denote by $\operatorname{Diff}^{+}(S)$ the group of orientation preserving diffeomorphisms of $S$ and by $\operatorname{Imm}_{f}(S, M)$ the set of all smooth free immersions $\iota: S \rightarrow M$, i.e., smooth immersions $\iota$ such that the stabilizer subgroup of $\iota$ in $\operatorname{Diff}^{+}(S)$ is trivial. For instance, if $\iota: S \rightarrow M$ is a somewhere injective immersion, i.e. there exists $x_{0} \in S$ such that $\iota^{-1}\left(\iota\left(x_{0}\right)\right)=x_{0}$, then $\iota \in \operatorname{Imm}_{f}(S, M)$ CMM1991, Lemma 1.4]. We will denote by $B_{i, f}^{+}(S, M)$ the quotient $\operatorname{Imm}_{f}(S, M) / \operatorname{Diff}^{+}(S)$. Note that if $S$ is connected, $\operatorname{Diff}^{+}(S)$ is an index two subgroup of the group $\operatorname{Diff}(S)$ of all diffeomorphisms of $S$, and therefore $B_{i, f}^{+}(S, M)$ is a double covering of $B_{i, f}(S, M):=\operatorname{Imm}_{f}(S, M) / \operatorname{Diff}(S)$. As a side remark we notice that this latter quotient is what is called a shape space in computer vision community [BBM2013, Michor2016].

Having introduced this notation, we can state our 
Theorem 1.3 (Main Theorem). Assume that a Riemannian manifold $(M, g)$ admits a parallel $r$-fold $V C P \chi \in \Omega^{r}(M, T M)$. Let $S$ be a closed oriented $(r-1)$-dimensional manifold. Then the space $B_{i, f}^{+}(S, M)$ has a structure of a formally Kähler manifold $\left(B_{i, f}^{+}(S, M), J, L_{2}(g), \omega^{2}\right)$ where $J$ is an almost complex structure with vanishing Nijenhuis tensor, $L_{2}(g)$ is the weak $L_{2}$-metric, and $\omega^{2}$ is the associated closed weak symplectic 2-form. Furthermore $J$ is parallel w.r.t. to the Levi-Civita connection $\nabla^{L C}$ of the weak metric $L_{2}(g)$.

Notation and conventions. Through the whole paper $S$ will always be a closed (i.e., compact without boundary) oriented finite dimensional manifold, $M$ a finite dimensional manifold and $(M, g)$ a finite dimensional Riemannian manifold, $\mathfrak{M}, \mathfrak{N}$ smooth manifolds modeled on convenient vector spaces, $\mathfrak{X}(\mathfrak{M})$ the space of smooth (kinematic) vector fields on $\mathfrak{M}$. For a smooth vector bundle $E$ over a smooth manifold $\mathfrak{M}$ we denote by $\Gamma(E)$ the space of all smooth sections of $E$. For smooth manifolds $\mathfrak{M}$ and $\mathfrak{N}$ we denote by $C^{\infty}(\mathfrak{M}, \mathfrak{N})$ the space of all smooth mappings from $\mathfrak{M}$ to $\mathfrak{N}$. If $\mathfrak{N}=\mathbb{R}$ we abbreviate $C^{\infty}(\mathfrak{M}, \mathbb{R})$ as $C^{\infty}(\mathfrak{M})$. For an Euclidean vector bundle $E \rightarrow M$ with a fiberwise metric $h$ and any $u, v \in \Gamma(E)$ we denote by $h(u, v)$ the smooth function on $M$ defined by $h(u, v)(x):=h(u(x), v(x))$ for all $x \in M$.

The paper is organized as follows. In the second section we collect some known results on geometry of the space $B_{i, f}^{+}(S, M)$ endowed with the weak $L_{2}$-metric. Then we prove one of our main technical results (Lemma 2.8), which is needed for our establishing the explicit formula of the Levi-Civita connection of the $L_{2}$-metric (Theorem 2.10) as well as for the proof of our Main Theorem. In the last section first we recall Lee-Leung's construction

of the almost complex structure on $B_{i, j}^{+}(S, M)$. Then we give a proof of the Main Theorem.

\section{Riemannian geometry of the space $B_{i, f}^{+}(S, M)$}

In this section, first we fix necessary notation and recall some known results on the Fréchet manifold structure of the moduli space $B_{i, f}^{+}(S, M)$. Then we give an explicit formula for the Levi-Civita connection of the weak $L_{2^{-}}$ metric on $B_{i, f}^{+}(S, M)$ (Theorem 2.10), generalizing to an arbitrary oriented submanifold $S$ results by Henrich for the cases $\operatorname{dim} S=1$ and $\operatorname{codim} S=1$ Henrich2009, Theorem 3.1, p. 25, Theorem 5.21, p. 48] The proof consists in showing that Henrich's connection $\nabla^{\perp}$ on the space $\operatorname{Imm}_{f}(S, M)$ Henrich2009, Definition 5.2, p. 41] is torsion-free independently of the dimension of $S$. In Henrich2009 a complete proof of the torsion-freeness of $\nabla^{\perp}$ is only given for the case $\operatorname{dim} S=1$ [Henrich2009, Lemma 2.17, p. 19], but this proof is supposedly immediately generalized/adapted to the general case (e.g., in Henrich2009, Definition 5.2, p. 41] the connection $\nabla^{\perp}$ is declared to be torsion-free without the need of a proof). Our proof of the 
torsion-freeness of $\nabla^{\perp}$ uses Lemma 2.8, which is possibly of independent interest and is employed further in the proof of the Main Theorem.

2.1. The smooth structure on $B_{i, f}^{+}(S, M)$. The space $B_{i, f}(S, M)$ has been considered by Cervera-Mascaró-Michor in [CMM1991, see also KM1997, 44.2 , p. 476] without the assumption that $S$ is a closed oriented manifold and in MM2005 with the assumption that $S$ is closed. They observed that the space $\operatorname{Imm}_{f}(S, M)$ is an open invariant subset in the space $C^{\infty}(S, M)$ of all smooth maps from $S$ to $M$ endowed with compact-open topology and the right action of $\operatorname{Diff}(S)$. If $S$ is a closed submanifold then any immersion $\iota: S \rightarrow M$ is proper. In this case, the results from the above mentioned papers imply the following statements (1)-(6).

(1) $\operatorname{Imm}_{f}(S, M)$ can be naturally endowed with the structure of an infinite dimensional smooth manifold modeled on the Fréchet space of smooth sections $\Gamma\left(\iota^{*} T M\right)$ along a smooth immersion $\iota: S \rightarrow M$, see e.g. KM1997, Theorem 42.1, p. 439].

(2) $\operatorname{Imm}_{f}(S, M)$ is the total space of a smooth principal fiber bundle $\pi: \operatorname{Imm}_{f}(S, M) \rightarrow B_{i, f}(S, M)$ whose fiber is $\operatorname{Diff}(S)$, and hence $\operatorname{Imm}_{f}(S, M)$ is also the total space of a smooth principal fiber bundle $\pi^{+}: \operatorname{Imm}_{f}(S, M) \rightarrow$ $B_{i, f}^{+}(S, M)$ whose fiber is $\operatorname{Diff}^{+}(S)$.

(3) $B_{i, f}(S, M)$ and $B_{i, f}^{+}(S, M)$ are Hausdorff spaces in the quotient topology.

(4) For an immersion $\iota: S \rightarrow M$ let $N_{\iota} S$ be the normal bundle $\iota^{*} T M / T S$, whose fiber at $s \in S$ can be identified with the orthogonal complement $T_{\iota(s)} \iota(S)^{\perp}$ of $T_{\iota(s)} \iota(S)$ in $T_{\iota(s)} M$ if $M$ is a Riemannian manifold. Then $B_{i, f}^{+}(S, M)$ inherits the structure of a smooth Fréchet manifold locally modeled on the Fréchet space $\Gamma\left(N_{\iota} S\right)$ of smooth sections of $N_{\iota} S$, which is identified with the kinematic tangent space $T_{[\iota]} B_{i, f}^{+}(S, M)$, where $[\iota]:=\pi^{+}(\iota)$.

(5) The kinematic tangent bundle $T B_{i, f}^{+}(S, M)$ has the structure of a smooth vector bundle over $B_{i, f}^{+}(S, M)$. The Lie bracket of two kinematic vector fields, i.e., of two smooth sections of $T B_{i, f}^{+}(S, M)$, is well-defined [KM1997, Theorem 32.8, p. 327].

(6) Let $E \rightarrow B_{i, f}^{+}(S, M)$ be a smooth vector bundle, whose fiber $F$ is a convenient vector space, e.g., $F=\mathbb{R}^{n}$. Then we denote by $L_{a l t}^{k}\left(T B_{i, f}^{+}(S, M), E\right)$ the smooth bundle over $B_{i, f}^{+}(S, M)$ whose fiber over $[\iota] \in B_{i, f}^{+}(S, M)$ consists of all alternating bounded $k$-linear maps from $T_{[\iota]} B_{i, f}^{+}(S, M) \times \cdots{ }_{k t i m e s} \times$ $T_{[\iota]} B_{i, f}^{+}(S, M)$ to $E_{[\iota]}$. We set

$$
\Omega^{k}\left(B_{i, f}^{+}(S, M), E\right):=\Gamma\left(L_{a l t}^{k}\left(T B_{i, f}^{+}(S, M), E\right)\right)
$$

and call it the space of differential forms on $B_{i, f}^{+}(S, M)$ with values in $E$ KM1997, $§ 33.22$, p. 352]. If $E=B_{i, f}^{+}(S, M) \times \mathbb{R}$ is the trivial vector 
bundle with fiber $\mathbb{R}$ over $B_{i, f}^{+}(S, M)$ then we abbreviate $\Omega^{k}\left(B_{i, f}^{+}(S, M), E\right)$ as $\Omega^{k}\left(B_{i, f}^{+}(S, M)\right)$.

From now on we shall omit the adjective "kinematic" before "tangent vector (field)".

Example 2.1. (cf. LL2007, Vizman2011) Let $\varphi \in \Omega^{k}(M)$ and $\operatorname{dim} S=r$. Denote by $p_{2}: S \times \operatorname{Imm}_{f}(S, M) \rightarrow \operatorname{Imm}_{f}(S, M)$ the projection onto the second factor. Since the evaluation map

$$
\begin{aligned}
e v: S \times \operatorname{Imm}_{f}(S, M) & \rightarrow M \\
(s, \iota) & \mapsto \iota(s)
\end{aligned}
$$

is smooth KM1997, Corollary 3.1.3(1), p. 31], for any $\varphi \in \Omega^{k}(M)$ we have $e v^{*}(\varphi) \in \Omega^{k}\left(S \times \operatorname{Imm}_{f}(S, M)\right)$. Next, using the integration over fiber, which is a differential version of the slant map, we can push $e v^{*}(\varphi)$ to a form $\left(p_{2}\right)_{*}(e v)^{*}(\varphi) \in \Omega^{k-r}\left(\operatorname{Imm}_{f}(S, M)\right)$. It is not hard to see that $\left(p_{2}\right)_{*}(e v)^{*}(\varphi)=\left(\pi^{+}\right)^{*}\left(\varphi_{B}\right)$ where $\varphi_{B} \in \Omega^{k-r}\left(B_{i, f}^{+}(S, M)\right)$ is defined by

$$
\varphi_{B}\left(X_{1}, \cdots, X_{k-r}\right):=\left.\int_{S} i_{X_{1}} \cdots i_{X_{k-r}} \varphi\right|_{S}
$$

for $X_{1}, \cdots, X_{k-r} \in \Gamma\left(N_{\iota} S\right)$ where $\left.\varphi\right|_{S} \in \Gamma\left(S, \wedge^{r} \iota^{*} T M^{*}\right)$ is the restriction of $\varphi$ to $S$. If $d \varphi=0$ then $d\left(p_{2}\right)_{*}(e v)^{*}(\varphi)=0$ and therefore $d \varphi_{B}=0$. The form $\varphi_{B}$ is called the transgression of $\varphi$.

Example 2.2. (cf. [LL2007, Vizman2011]) Let $\varphi \in \Omega^{k}(M, T M)$ and let $\operatorname{dim} S=r$. For any immersion $\iota: S \rightarrow M$, let $\operatorname{vol}_{\iota^{*} g}$ be the volume form of $S$ associated with the induced metric $\iota^{*} g$, and let $\overrightarrow{T_{s} S} \vec{\imath}$ denote the unit $r$-vector of $T_{s} S$ with respect to this volum form. Let $\Pi: T_{\iota(s)} M \rightarrow N_{\iota(s)} S$ be the natural projection. Then we define the form $\left.\varphi_{B} \in \Omega^{k-r}\left(B_{i, f}^{+} S, M\right), T B_{i, f}^{+}(S, M)\right)$ by

$$
\varphi_{B}\left(X_{1}, \cdots, X_{k-r}\right)(s):=\Pi\left(i_{X_{1}} \cdots i_{X_{k-r}}\left(\left.i_{T_{s} S_{\iota}} \varphi\right|_{S}\right)\right)
$$

for $X_{1}, \cdots, X_{k-r} \in \Gamma\left(N_{\iota} S\right)$. The form $\varphi_{B}$ is called the transgression of $\varphi$ defined by the induced volume form on $S$.

2.2. The $L_{2}$-Riemannian metric and its Levi-Civita connection on $B_{i, f}^{+}(S, M)$. In this subsection we assume that $(M, g)$ is a Riemannian manifold. It is known that the space $B_{i, f}^{+}(S, M)$ is endowed with the weak $L_{2^{-}}$ Riemmannian metric defined as follows

$$
\langle u, v\rangle_{[\iota]}=\int_{S} g(u, v) \operatorname{vol}_{\iota * g}
$$

where $u, v \in \Gamma\left(N_{\iota} S\right)=T_{[\iota]} B_{i, f}^{+}(S, M)$. The RHS of (2.3) extends naturally to a $L_{2}$-metric on the space $\operatorname{Imm}_{f}(S, M)$, which is invariant under the action of $\operatorname{Diff}^{+}(S)$ and therefore it descends to the $L_{2}$-metric on $B_{i, f}^{+}(S, M)$ 
making the projection $\pi^{+}: \operatorname{Imm}_{f}(S, M) \rightarrow B_{i, f}^{+}(S, M)$ a Riemannian submersion. Kainz proved the existence of the Levi-Civita connection for the $L_{2}$-metric on the space $\operatorname{Imm}_{f}(S, M)$ Kainz1984, Theorem 2.2]. Since the $L_{2}$-metric on $\operatorname{Imm}_{f}(S, M)$ is $\operatorname{Diff}^{+}(S)$-invariant, the associated Levi-Civita connection is also $\operatorname{Diff}^{+}(S)$-invariant. A formula for the Levi-Civita connection on $B_{i, f}^{+}(S, M)$ has been given by Michor and Mumford via the equation for geodesics on $B_{i, f}^{+}(S, M)$ [MM2005, §4.2] and explicitly by Henrich in his $\mathrm{PhD}$ Thesis for the case $\operatorname{dim} S=1$ or $\operatorname{codim} S=1$ Henrich2009, Theorem 3.1, Theorem 5.21]. In the remaining part of this subsection we shall generalize Henrich's formula for an arbitrary closed oriented manifold $S$.

First we recall the notion of an affine connection and its associated covariant differentiation on a smooth possibly infinite dimensional manifold $\mathfrak{M}$ modelled on a convenient vector space, following [KM1997, MMM2013, and Kainz1984. Denote by $\mathfrak{X}(\mathfrak{M})$ the space of smooth vector fields on $\mathfrak{M}$.

Definition 2.3. An affine connection on a smooth manifold $\mathfrak{M}$ is a smooth mapping

$$
\nabla: \mathfrak{X}(\mathfrak{M}) \times \mathfrak{X}(\mathfrak{M}) \rightarrow \mathfrak{X}(\mathfrak{M})
$$

which is a derivation on the second argument and $C^{\infty}(\mathfrak{M})$-linear on the first argument. In other words, re-denoting $\nabla(X, Y)$ as $\nabla_{X}(Y)$, we have

$$
\begin{array}{r}
\nabla_{X}(Y+Z)=\nabla_{X}(Y)+\nabla_{X}(Z) \\
\nabla_{X}(f Y)=f \nabla_{X}(Y)+(X f) Y \\
\nabla_{f X+g Y}(Z)=f \nabla_{X} Y+g \nabla_{Y} Z
\end{array}
$$

for all $X, Y, Z \in \mathfrak{X}(\mathfrak{M})$ and $f, g \in C^{\infty}(\mathfrak{M})$. The operator $\nabla_{X}$ is called a covariant differentiation w.r.t. $X$. If the manifold $\mathfrak{M}$ is equipped with a Riemannian metric

$$
\langle,\rangle: \mathfrak{X}(\mathfrak{M}) \times \mathfrak{X}(\mathfrak{M}) \rightarrow C^{\infty}(\mathfrak{M}),
$$

then the covariant derivation is called Levi-Civita, if it respects the metric and it is torsion-free, i.e.,

$$
\begin{array}{r}
X\langle Y, Z\rangle=\left\langle\nabla_{X} Y, Z\right\rangle+\left\langle Y, \nabla_{X} Z\right\rangle \\
T^{\nabla}(X, Y):=\nabla_{X} Y-\nabla_{Y} X-[X, Y]=0 .
\end{array}
$$

As in the finite dimensional case, the above conditions determine $\nabla$ uniquely, so that, when it exists we talk of the Levi-Civita connection.

Remark 2.4. It is not hard to see that the above definition of an affine connection on $\mathfrak{M}$ is equivalent to the definition of an affine connection via a smooth projection $K: T^{2} \mathfrak{M} \rightarrow T \mathfrak{M}$ KM1997, Definition 37.2, p. 376].

Furthermore, the notions of an affine connection and of the associated covariant dfferentiation on $\mathfrak{M}$ are particular cases of those of a linear connection on a smooth vector bundle $E$ over $\mathfrak{M}$ and of its associated covariant differentiation $\nabla: \mathfrak{X}(\mathfrak{M}) \times \Gamma(E) \rightarrow \Gamma(E)$ KM1997, 37.27, 37.28, p. 397]. 
Remark 2.5. As $(X, Y) \mapsto \nabla_{X} Y$ is $C^{\infty}(\mathfrak{M})$-linear in the first variable, the datum of a covariant derivative $\nabla$ is equivalent to the datum of all the derivatives

$$
\nabla_{u}: \mathfrak{X}(\mathfrak{M}) \rightarrow T_{p} \mathfrak{M}
$$

with $u \in T_{p} \mathfrak{M}$ ranging in the tangent space of $\mathfrak{M}$ at $p$, with $p$ varying over the whole of $\mathfrak{M}$.

Remark 2.6. The torsion of an affine connection is a tensor, i.e., the value $T^{\nabla}(X, Y)_{p}$ of the vector field $T^{\nabla}(X, Y)$ at the point $p$ of $\mathfrak{M}$ only depends on the values $X_{p}$ and $Y_{p}$ of $X$ and $Y$ at $p$. In other words, one has a well defined bilinear map $u \otimes v \mapsto T_{p}^{\nabla}(u, v)$ defined by

$$
T_{p}^{\nabla}(u, v)=T^{\nabla}(X, Y)_{p}
$$

where $X$ and $Y$ are arbitrary extensions of the tangent vectors $u$ and $v$ at the point $p$, respectively, to tangent vector fields on a 2 -dimensional surface in $\mathfrak{M}$.

Next, following MM2005, cf. Henrich2009], we shall express the LeviCivita connection $\nabla^{L C}$ on $B_{i, f}^{+}(S, M)$ w.r.t. the $L_{2}$-metric as a sum of a torsion-free affine connection $\nabla^{\perp}$ on $B_{i, f}^{+}(S, M)$ and a symmetric tensor $\mathfrak{B}$. By Remark 2.5, to define $\nabla^{\perp}$ it suffices to define $\nabla_{u}^{\perp}$ for every tangent vector $u$ in $T_{[\iota]} B_{i, f}^{+}(S, M)$. Let $\gamma: \mathbb{R} \rightarrow \operatorname{Imm}_{f}(S, M)$ be a path of free immersions with $\gamma(0)=\iota$ whose velocity vector $\left.\dot{\gamma}\right|_{t=0}$ at $t=0$ is a lift/a representative of the tangent vector $u$ in $\Gamma\left(\iota^{*} T M\right)$. For all $k \in \mathbb{N}$ we have isomorphisms

$$
C^{\infty}\left(\mathbb{R}^{k}, C^{\infty}(S, M)\right)=C^{\infty}\left(\mathbb{R}^{k} \times S, M\right) .
$$

By means of this for $k=1$, we identify $\gamma$ with a map, which we will denote by the same symbol, $\gamma: \mathbb{R} \times S \rightarrow M$, KM1997, Corollary 3.13, p. 31]. At any point $s$ of $S, \gamma(\cdot, s)$ defines a curve in $M$ whose velocity vector $u(s)$ at $t=0$ is a vector in $T_{\gamma(0, s)} M$. For any vector field $X$ on $B_{i, f}^{+}(S, M)$, and for any fixed $t$, the vector $X_{[\gamma(t)]}$ can be identified with a section of the normal bundle $N_{\gamma(t)} S$. By identifying $N_{\gamma(t)} S$ with a subbundle of the tangent bundle $(\gamma(t))^{*} T M$, e.g., by means of the Riemannian metric as in Subsection 2.1 (4), we can think of $X_{[\gamma]}:=X \circ \gamma$ as a section of $\gamma^{*} T M$. The pullback of the Levi-Civita connection on $T M$ gives a connection $\gamma^{*}\left(\nabla^{L C ; M}\right)$ on $\gamma^{*} T M$ and we can define

$$
\left(\nabla_{u}^{\perp} X\right):=\left(\left.\gamma^{*}\left(\nabla^{L C ; M}\right)_{\frac{\partial}{\partial t}} X_{[\gamma]}\right|_{t=0}\right)^{\perp}
$$

where on the right hand side $(-)^{\perp}$ denotes its projection on the normal bundle $N_{[\iota]} S$.

Proposition 2.7. (cf. [Henrich2009, Lemma 2.17]) The formula (2.10) defines a torsion-free connection on $B_{i, f}^{+}(S, M)$.

Proof. One immediately sees that (2.10) defines an affine connection, so we are only left with showing that the torsion $T^{\perp}(u, v)$ of $\nabla^{\perp}$ vanishes for any 
$u, v \in T_{[\iota]} B_{i, f}^{+}(S, M)=N_{\iota} S$. To do this we shall use the following argument, which shall be utilized several times in our paper.

We consider a deformation

$$
\gamma:(-\varepsilon, \varepsilon) \times(-\varepsilon, \varepsilon) \times S \rightarrow M,(x, y, s) \mapsto \gamma(x, y, s),
$$

such that for all $s \in S$,

$$
\gamma(0,0, s)=\iota(s),\left.\quad \frac{d \gamma}{d x}\right|_{(0,0, s)}=u(s), \quad \text { and }\left.\quad \frac{d \gamma}{d y}\right|_{(0,0, s)}=v(s) .
$$

More explicitly we set

$$
\gamma(x, y, s):=\operatorname{Exp}_{\iota(s)}(x \cdot u(s)+y \cdot v(s)),
$$

where $\operatorname{Exp}_{\iota(s)}: T_{\iota(s)} M \rightarrow M$ is the exponential map for the Riemannian manifold $M$ at the point $\iota(s)$. This deformation extends the vectors $u, v \in$ $T_{[\iota]} B_{i, f}^{+}(S, M)=N_{\iota} S$ to vector fields, that we denote by $X$ and $Y$, along the image of $\gamma((-\varepsilon, \varepsilon) \times(-\varepsilon, \varepsilon))$ in $\operatorname{Imm}_{f}(S, M)$ via the isomorphism (2.9), i.e., the vector field $X$ is defined by

$$
X_{\gamma(x, y)}(s)=\left.\left(d \operatorname{Exp}_{\iota(s)}\right)\right|_{x u(s)+y v(s)}(u(s))
$$

and similarly for $Y$. The projection $\pi^{+}: \operatorname{Imm}_{f}(S, M) \rightarrow B_{i, f}^{+}(S, M)$ maps $X, Y$ to vector fields $X^{\mathrm{ver}}, Y^{\mathrm{ver}}$ along the image of $\pi^{+} \circ \gamma((-\varepsilon, \varepsilon) \times(-\varepsilon, \varepsilon))$. By definition we can identify $X^{\mathrm{ver}}\left(\pi^{+} \circ \gamma(x, y)\right)(s)$ with the projection of $X(s)$ on the normal bundle $N_{\gamma(x, y, s)} S$, i.e., we have the following decomposition

$$
X(\gamma(x, y))=X^{\mathrm{ver}}\left(\pi^{+} \circ \gamma(x, y)\right)+X^{\mathrm{hor}}\left(\pi^{+} \circ \gamma(x, y)\right),
$$

where $X^{\text {hor }}\left(\pi^{+} \circ \gamma(x, y)\right) \in \mathfrak{X}(\gamma(x, y) S)$. By construction, the vector fields $X^{\mathrm{ver}}, Y^{\mathrm{ver}}$ extend the tangent vectors $u, v$, so the torsion $T^{\perp}(u, v)$ is computed by $T^{\perp}(u, v)=\left(\nabla_{X}^{\perp}{ }^{\text {ver }} Y^{\text {ver }}-\nabla_{Y^{\text {ver }}}^{\perp} X^{\text {ver }}-\left[X^{\text {ver }}, Y^{\text {ver }}\right)_{[\iota]}\right.$. Since $X^{\text {ver }}=$ $\left(\pi^{+}\right)_{*} X$ and $[X, Y]=0$, we have $\left[X^{\mathrm{ver}}, Y^{\mathrm{ver}}\right]=0$. The conclusion of the proof is then immediate from the following Lemma 2.8 .

Lemma 2.8. Assume that $X^{\mathrm{ver}}, Y^{\mathrm{ver}}$ are generated by $\gamma$ defined in (2.11) and (2.13). Then we have

$$
\nabla_{X}^{\mathrm{ver}} Y_{[\iota]}^{\mathrm{ver}}=\nabla_{Y^{\mathrm{ver}}}^{\perp} X_{[\iota]}^{\mathrm{ver}}=0 .
$$

Proof. As the statement is symmetric in $X^{\mathrm{ver}}$ and $Y^{\mathrm{ver}}$, we only need to show that $\nabla_{X^{\text {ver }}}^{\perp} Y_{[\iota]}^{\text {ver }}=0$. To prove Lemma 2.8, using (2.10), it suffices to show that for any $w \in \Gamma\left(N_{\iota} S\right)$ and any $s \in S$ we have

$$
g\left(\left.\gamma^{*}\left(\nabla^{L C ; M}\right)_{\frac{\partial}{\partial x}} Y_{[\gamma]}^{\mathrm{ver}}\right|_{(x, y)=(0,0)}, w\right)_{s}=0 .
$$

Since $\gamma$ is a restriction of the exponential map $\operatorname{Exp}: T M \rightarrow M$ at $\iota(S)$, we have

$$
\left.\gamma^{*}\left(\nabla^{L C ; M}\right)_{\frac{\partial}{\partial x}} Y_{[\gamma]}\right|_{(x, y)=(0,0)}=0 .
$$

Using 2.16, to prove (2.15) it suffices to show that 


$$
g\left(\left.\gamma^{*}\left(\nabla^{L C ; M}\right) \frac{\partial}{\partial x} Y_{[\gamma]}^{\mathrm{hor}}\right|_{(x, y)=(0,0)}, w\right)_{s}=0 .
$$

Abusing the same notation $\gamma$, now we let $\gamma$ be a map from $(-\varepsilon, \varepsilon) \times(-\varepsilon, \varepsilon) \times$ $(-\varepsilon, \varepsilon) \times S \rightarrow M$ defined by (cf. (2.11) $)$

$$
\gamma(x, y, z, s):=\operatorname{Exp}_{\iota(s)}(x \cdot u(s)+y \cdot v(s)+z \cdot w(s)),
$$

and let $Z$ be the vector field along the image of $\gamma$ defined in analogy to $X$ and $Y$. Then, as in (2.16), we have

$$
\left.\gamma^{*}\left(\nabla^{L C ; M}\right)_{\frac{\partial}{\partial x}} Z_{[\gamma]}\right|_{(x, y, z)=(0,0,0)}=0 .
$$

and so equation (2.17) is equivalent to

$$
\gamma^{*}\left(\nabla^{L C ; M}\right)_{\frac{\partial}{\partial x}} g\left(Y_{[\gamma]}^{\mathrm{hor}}, Z_{[\gamma]}\right)_{\mid(x, y, z)=(0,0,0)}=0 .
$$

Noting that $g\left(Y^{\text {hor }}, Z^{\text {ver }}\right)=0$, to prove (2.17) it suffices to show that

$$
\gamma^{*}\left(\nabla^{L C ; M}\right)_{\frac{\partial}{\partial x}} g\left(Y_{[\gamma]}^{\text {hor }}, Z_{[\gamma]}^{\text {hor }}\right)_{\mid(x, y, z)=(0,0,0)}=0 .
$$

Since $Y \circ \gamma:(-\epsilon, \epsilon)^{3} \times S \rightarrow T M$ is a differentiable map, the composition $Y^{\text {hor }} \circ \gamma:(-\varepsilon, \varepsilon)^{3} \times S \rightarrow T M$ is a differentiable map. Since $Y^{\text {hor }} \circ$ $\gamma(0,0,0, s)=0$, we can write for $x \in[-\varepsilon / 2, \varepsilon / 2]$

$$
Y^{\text {hor }} \circ \gamma(x, 0,0, s)=x \cdot Y^{\prime}(x, 0,0, s)
$$

where $Y^{\prime}(x, 0,0, s) \in T_{\gamma(x, 0,0, s)} M$ and $\left|Y^{\prime}(x, 0,0, s)\right| \leqslant A(s)$ for some positive number $A(s) \in \mathbb{R}$. Since $S$ is compact, we can choose $A(s)$ independent of $s$. The same argument applies to $Z \circ \gamma$. This yields (2.21) immediately and completes the proof of Lemma 2.8

Next we shall define the desired symmetric tensor $\mathfrak{B}$. As $(M, g)$ is a Riemannian manifold and $S$ is oriented, we have a volume form map

$$
\begin{aligned}
\operatorname{vol}_{S}: \operatorname{Imm}_{i, f}(S, M) & \rightarrow \Omega^{\operatorname{dim} S}(S ; \mathbb{R}) \\
\iota & \mapsto \operatorname{vol}_{\iota * g},
\end{aligned}
$$

where $\operatorname{vol}_{L^{*} g} \in \Omega^{\operatorname{dim} S}(S ; \mathbb{R})$ denotes the volume form on $S$ associated with the pullback metric $\iota^{*} g$. As $\operatorname{vol}_{S}$ is a smooth function on $\operatorname{Imm}_{i, f}(S, M)$ with values in a vector space, we can take its derivative $X \operatorname{vol}_{S}$ with respect to a smooth vector field on $\operatorname{Imm}_{i, f}(S, M)$ and this will again be a smooth $\Omega^{\operatorname{dim} S}(S ; \mathbb{R})$-valued function on $\operatorname{Imm}_{i, f}(S, M)$. If $\tilde{\iota}=\iota \circ \phi$, then $\phi:\left(S, \iota^{*} g\right) \rightarrow$ $\left(S, \tilde{\iota}^{*} g\right)$ is an isometry. This implies that the equation

$$
g\left(X_{[\iota]}, W_{[\iota]}\right)\left(\operatorname{vol}_{S}\right)_{[\iota]}=\left(X \operatorname{vol}_{S}\right)_{[\iota]}
$$

for any vector field $X$ on $B_{i, f}^{+}(S, M)$, is well-defined. Namely, the equivariance conditions $\left(\operatorname{vol}_{S}\right)_{\tilde{\iota}}=\phi^{*}\left(\left(\operatorname{vol}_{S}\right)_{\iota}\right)$ and $X_{\tilde{\iota}}=\phi^{*} X_{\iota}$ imply that a solution $W$ is $\operatorname{Diff}^{+}(S)$-equivariant as well. 
By the very definition of the mean curvature vector field for an immersed submanifold we have the following.

Lemma 2.9. The equation 2.23 has a unique solution $W_{[\iota]}=-(\operatorname{dim} S) H_{\iota(S)}$, where $H_{\iota(S)}$ is the mean curvature of the immersed submanifold $\iota(S)$, defined by

$$
H_{\iota(S)}=\frac{1}{\operatorname{dim} S} \sum_{i=1}^{\operatorname{dim} S}\left(\nabla_{e_{i}}^{L C ; M} e_{i}\right)^{\perp},
$$

where the $e_{i}$ are orthonornal tangent vector fields on a neighborhhod of a point $s$ in $S$ and $\perp$ denotes the projection on the normal bundle. The solution $W_{[\iota]}=-(\operatorname{dim} S) H_{\iota(S)}$ defines a vector field $W$ on $B_{i, f}^{+}(S, M)$.

Consider now the bilinear form

$$
\mathfrak{B}: T B_{i, f}^{+}(S, M) \times T B_{i, f}^{+}(S, M) \rightarrow T B_{i, f}^{+}(S, M)
$$

defined by

$$
\mathfrak{B}(u, v)_{[\iota]}=g(u, v) W_{[\iota]}-g\left(u, W_{[\iota]}\right) v-g\left(v, W_{[\iota]}\right) u
$$

for any $u, v \in T_{[\iota]} B_{i, f}^{+}(S, M)$, where $W$ is the multiple of the mean curvature vector field defined in Lemma 2.9, and where we use the $C^{\infty}(S ; \mathbb{R})$-module structure on $T_{[\iota]} B_{i, f}^{+}(S, M)=N_{\iota} S$.

The following theorem generalizes to an arbitrary $S$ results by Henrich for the cases $\operatorname{dim} S=1$ and $\operatorname{codim} S=1$ Henrich2009, Theorem 3.1, p. 25, Theorem 5.21, p. 50].

Theorem 2.10. The covariant derivation $\nabla^{\perp}-\frac{1}{2} \mathfrak{B}$ is the Levi-Civita covariant derivation on $B_{i, f}^{+}(S, M)$.

Proof. By uniqueness, we only need to show that $\nabla^{\perp}-\frac{1}{2} \mathfrak{B}$ is torsion-free and compatible with the Riemannian metric on $B_{i, f}^{+}(S, M)$. The torsionfreeness is immediate, as $\nabla^{\perp}$ is torsion-free, and $\mathfrak{B}$ is a symmetric bilinear form. Next, we show that

$$
X\langle Y, Z\rangle=\left\langle\nabla_{X}^{\perp} Y, Z\right\rangle+\left\langle Y, \nabla_{X}^{\perp} Z\right\rangle+-\frac{1}{2}(\langle\mathfrak{B}(X, Y), Z\rangle+\langle Y, \mathfrak{B}(X, Z)\rangle)
$$

holds for any $X, Y, Z \in \mathfrak{X}\left(B_{i, f}^{+}(S, M)\right)$. As the difference between the left and the right hand side of the above equation is a tensor, without loss of generality we can assume that $X, Y, Z$ are generated by a three-parameter variation $\bar{\gamma}: \mathbb{R}^{3} \rightarrow B_{i, f}^{+}(S, M)$ with $\bar{\gamma}(0,0,0)=[\iota]$, e.g., as in (2.18). The map $\bar{\gamma}$ lifts to a smooth map $\gamma:(-\epsilon, \epsilon)^{3} \rightarrow \operatorname{Imm}_{f}(S, M)$ defined by a smooth map

$$
\begin{aligned}
\gamma:(-\epsilon, \epsilon) \times(-\epsilon, \epsilon) \times(-\epsilon, \epsilon) \times S & \rightarrow M, \\
(x, y, x, s) & \mapsto \gamma(x, y, z, s),
\end{aligned}
$$


with $\gamma(0,0,0, s)=\iota(s)$ and such that $\gamma$ is purely normal at $(x, y, z)=$ $(0,0,0)$. Then we can assume at every $(x, y, z)$ we have

$$
X=\left(\frac{d \gamma}{d x}\right)^{\mathrm{ver}}, Y=\left(\frac{d \gamma}{d y}\right)^{\mathrm{ver}}, Z=\left(\frac{d \gamma}{d x}\right)^{\mathrm{ver}}
$$

and find

$$
\begin{aligned}
(X\langle Y, Z\rangle)_{[\iota]} & =\left.\frac{d}{d x} \int_{S} g\left(Y_{[\gamma]}, Z_{[\gamma]}\right)\left(\operatorname{vol}_{S}\right)_{\gamma}\right|_{(x, y, z)=(0,0,0)}+\left.\int_{S} g\left(Y_{[\gamma]}, Z_{[\gamma]}\right)\left(\frac{d}{d x}\left(\operatorname{vol}_{S}\right)_{\gamma}\right)\right|_{(x, y, z)=(0,0,0)} \\
& =\left.\int_{S}\left(\frac{d}{d x} g\left(Y_{[\gamma]}, Z_{[\gamma]}\right)\right)\left(\operatorname{vol}_{S}\right)_{\gamma}\right|_{(x, y, z)=(0,0,0)} \\
& \left.\left.=\int_{S}\left(g\left(\gamma^{*}\left(\nabla^{L C ; M}\right)_{\frac{\partial}{\partial x}} Y_{[\gamma]}\right), Z_{[\gamma]}\right)+g\left(Y_{[\gamma]}, \gamma^{*}\left(\nabla^{L C ; M}\right)_{\frac{\partial}{\partial x}} Z_{[\gamma]}\right)\right)\right)\left.\left(\operatorname{vol}_{S}\right)_{\gamma}\right|_{(x, y, z)=(0,0,0)} \\
& \quad+\left.\int_{S} g\left(Y_{[\gamma]}, Z_{[\gamma]}\right)\left(\frac{d}{d x}\left(\operatorname{vol}_{S}\right)_{\gamma}\right)\right|_{(x, y, z)=(0,0,0)} \\
= & \int_{S}\left(g\left(\left(\nabla_{X}^{\perp} Y\right)_{[\iota]}, Z_{[\iota]}\right)+g\left(Y_{[\iota]},\left(\nabla_{X}^{\perp} Z\right)_{[\iota]}\right)\left(\operatorname{vol}_{S}\right)_{[\iota]}+\int_{S} g\left(Y_{[\iota]}, Z_{[\iota]}\right)\left(X \operatorname{vol}_{S}\right)_{[\iota]}\right. \\
= & \left\langle\nabla_{X}^{\perp} Y, Z\right\rangle_{[\iota]}+\left\langle Y, \nabla_{X}^{\perp} Z\right\rangle_{[\iota]}+\int_{S} g\left(X_{[\iota]}, W_{[\iota]}\right) g\left(Y_{[\iota]}, Z_{[\iota]}\right)\left(\operatorname{vol}_{S}\right)_{[\iota]} .
\end{aligned}
$$

In the above equation we used that, as $Z_{[\iota]}=Z_{[\iota]}^{\mathrm{ver}}$, we have

$$
\begin{aligned}
\left.g\left(\gamma^{*}\left(\nabla^{L C ; M}\right) \frac{\partial}{\partial x} Y_{[\gamma]}\right), Z_{[\gamma]}\right)\left.\right|_{(x, y, z)=(0,0,0)} & \left.=g\left(\left(\gamma^{*}\left(\nabla^{L C ; M}\right)_{\frac{\partial}{\partial x}} Y_{[\gamma]}\right)\right)^{\perp}, Z_{[\gamma]}\right)\left.\right|_{(x, y, z)=(0,0,0)} \\
& =g\left(\left(\nabla_{X}^{\perp} Y\right)_{[\iota]}, Z_{[\iota]}\right) .
\end{aligned}
$$

Therefore, we are reduced to showing that

$$
\int_{S} g\left(X_{[\iota]}, W_{[\iota]}\right) g\left(Y_{[\iota]}, Z_{[\iota]}\right)\left(\operatorname{vol}_{S}\right)_{[\iota]}=-\frac{\langle\mathfrak{B}(X, Y), Z\rangle+\langle Y, \mathfrak{B}(X, Z)\rangle}{2},
$$

which is straightforward from the definition of $\mathfrak{B}$.

Remark 2.11. In [MMM2013, (8)], see also [Michor2016, §4.2], Micheli, Michor, and Mumford give more generally an existence condition for the Levi-Civita covariant derivative on a smooth manifold $M$ modeled on convenient locally convex vector spaces and endowed with a weak Riemannian metric $g$, i.e. a symmetric positive definite bilinear form $g$ such that $g_{x}^{b}: T_{x} M \rightarrow T_{x}^{*} M$ is only injective for each $x \in M$, in terms of symmetric gradients with respect to $g$.

\section{The Formally KähleR StRUCTURE OF HigheR Dimensional KNot SPACES}

In this section we give a proof of our Main Theorem 1.3. First we recall Lee-Leung's construction of the almost complex structure $J$ on $B_{i, f}^{+}(S, M)$ 
associated with a VCP on $M$. Then we derive Theorem 1.3 from Corollary 3.4 and Proposition 3.5 .

Denote by $G r^{+}(r, M)$ the Grassmanian bundle over $(M, g)$ whose fiber over $p \in M$ consists of the Grassmanian manifold of oriented $r$-dimensional subspaces in $T_{p} M$. For $v \in G r_{p}^{+}(r, M)$ let us denote by $\vec{v}$ the oriented unit $r$-vector associated to $v$ and to the Riemannian metric $g$ of $M$, and by $v^{\perp}$ the oriented orthogonal complement to $v$ in $T_{p} M$. Lee-Leung made the following simple but crucial observation [LL2007, p. 146]

Lemma 3.1. Let $\chi \in \Omega^{r+1}(M, T M)$ be a $V C P$. Then for any $v \in G r_{p}^{+}(r, M)$ and any $\xi \in v^{\perp} \subset T_{p} M$ we have

$$
i \vec{v} \chi_{p}(\xi) \in v^{\perp}
$$

Furthermore, the restriction of $i \vec{v} \chi_{p}$ to $v^{\perp}$ is a 1 -fold $V C P$ on $v^{\perp}$, denoted by $J(\chi, v)$, which satisfies the following relation for any $\xi, \zeta \in v^{\perp}$

$$
i \vec{v}\left(\varphi_{\chi}\right)_{p}(\xi, \zeta)=g_{p}(J(\chi, v) \xi, \zeta),
$$

where $\varphi_{\chi}$ is the VPC-form of $\chi$ (equation (1.1)).

We observe that Lemma 3.1 is a consequence of Gray's theorem Gray1969, Theorem 2.6].

Remark 3.2. As $1-\mathrm{VCP}$ on Euclidean vector spaces are equivalent to linear Kähler structures, one is naturally led to considering the Hermitian vector bundle $E(\chi)$ over $G r^{+}(r, M)$ whose fiber over $v$ is $v^{\perp}$ with the Hermitian structure $(g, J(\chi, v))$. As Chern classes are deformation invariants of the complex structure on a real vector bundle, the Chern classes of $E(\chi)$ are deformation invariants of the VCP structure $\chi$ on $M$. A detailed investigation of these and other deformation invariants of a VCP structure with emphasis on $G_{2^{-}}$and torsion-free $\operatorname{Spin}(7)$-manifolds, as well as a comparison with known deformation invariants of $G_{2}$-structures [CGN2018] will appear elsewhere.

Assume the dimension of the closed oriented manifold $S$ is $\operatorname{dim}_{\mathbb{R}} S=r$. At each point $(s, \iota)$ in $S \times \operatorname{Imm}_{f}(S, M)$, the image $\iota_{*} T_{s} S$ of the tangent space $T_{s} S$ in $T_{\iota(s)} M$ defines an element in $G r^{+}(r, M)$, so we have defined a smooth map

$$
v: S \times \operatorname{Imm}_{f}(S, M) \rightarrow G r^{+}(r, M) .
$$

By construction, $v^{\perp}(s, \iota)=N_{\iota(s)} S$, so by Lemma 3.1 we have a pointwise 1-VCP on the fibres of the normal bundle to $S$ at $\iota$, compatible with the inner product induced from $M$. From this it follows that we have the linear operator $J$ on the tangent bundle $T B_{i, f}^{+}(S, M)$ defined by setting, at each point $[\iota: S \rightarrow M]$,

$$
\left(J_{[\iota]} X\right)_{s}:=i_{T_{\iota(s)} S} \chi_{\iota(s)}\left(X_{s}\right)
$$


Lee and Leung observed that and $J$ is an almost complex structure on $T B(S, M)$ [L2007, p. 146], compatible with the $L_{2}$-metric, thus endowing $B_{i, f}^{+}(S, M)$ with the structure of an almost Hermitian manifold. As the fundamental 2-form of this Hermitian structure is the transgression defined by (2.2) of the $(r+2)$-VCP-form $\varphi_{\chi}$ defined in (1.1), [LL2007, Lemma 7], one sees that if $\varphi_{\chi}$ is closed, then $B_{i, f}^{+}(S, M)$ is an almost Kähler manifold.

In the remaining part of this section we assume that $\chi$ is a parallel VCP on $(M, g)$. In particular, this implies that $\varphi_{\chi}$ is closed.

Proposition 3.3. The almost complex structure $J$ is parallel with respect to the affine connection $\nabla^{\perp}$ of equation (2.10), i.e. $\nabla^{\perp} J=0$. Equivalently, $\nabla^{\perp}$ is an almost complex connection with respect to the almost complex structure $J$.

Proof. We need to prove that $\nabla_{X}^{\perp} J=0$ for any vector field $X$ on $B_{i, f}^{+}(S, M)$. As $\left(\nabla_{X}^{\perp} J\right) Y=\nabla_{X}^{\perp}(J Y)-J\left(\nabla^{\perp} Y\right)$, this is equivalent to proving

$$
\left\langle\nabla_{X}^{\perp}(J Y)-J\left(\nabla_{X}^{\perp} Y\right), Z\right\rangle_{[\iota]}=0
$$

for any three vector fields $X, Y, Z$ on $B_{i, f}^{+}(S, M)$, and any point $[\iota] \in B_{i, f}^{+}(S, M)$. Since the left hand side of (3.3) is a tensor, without loss of generality, as in the proof of Theorem 2.10 , we assume that $X, Y, Z$ are the vertical vector fields generated by the three-parameter variation

$$
\begin{aligned}
\gamma:(-\epsilon, \epsilon) \times(-\epsilon, \epsilon) \times(-\epsilon, \epsilon) \times S & \rightarrow M \\
(x, y, z, s) & \mapsto \gamma(x, y, z, s), \\
& \gamma(0,0,0, s)=\iota(s)
\end{aligned}
$$

defined by (2.18) and therefore we can apply Lemma 2.8, giving $\nabla_{X}^{\perp} Y=0$. From the defining equation (2.18), the restrictions of the vertical vector fields $X, Y$ and $Z$ at $(x, y, z)=(0,0,0)$ are the sections $u, v$ and $w$ of $N_{\iota} S$, respectively. We are then reduced to proving that

$$
\left\langle\nabla_{X}^{\perp}(J Y), Z\right\rangle_{[\iota]}=0
$$

i.e., due to fact that by (2.10) the value $\nabla_{X}^{\perp}(J Y)_{\iota(s)}$ depends only on $u(s)$ and due to the arbitrariness of the restriction $w$ of $Z$ at $[\iota]$, to show that

$$
g\left(\nabla_{u}^{\perp}(J Y), w\right)_{\iota(s)}=0
$$

for $Y$ the vertical vector field defined by (2.18), for any $u, w \in \Gamma\left(N_{\iota} S\right)$ and for all $s \in S$, which we prove next.

From the definition of $\nabla^{\perp}$, equation (2.10), and the definition of $J$, equation (3.2), we get

$$
g\left(\nabla_{u}^{\perp}(J Y), w\right)_{\iota(s)}=g\left(\left(\gamma^{*}\left(\nabla^{L C ; M}\right)_{\frac{\partial}{\partial x}} \chi\left(\overrightarrow{T_{\gamma(x, s)} \gamma(x)(S)}, Y\right)\right)^{\perp}, w\right)_{\iota(s)} .
$$


As $\chi$ is parallel with respect to the Levi-Civita connection on $M$, and since $w \in \Gamma\left(N_{\iota} S\right)$, we get

$$
\begin{gathered}
g\left(\nabla_{u}^{\perp}(J Y), w\right)_{\iota(s)}=g\left(\gamma^{*}\left(\nabla^{L C ; M}\right)_{\frac{\partial}{\partial x}} \chi\left(\overrightarrow{T_{[\gamma]} S}, Y\right), w\right)_{\iota(s)} \\
=g\left(\chi\left(\gamma^{*}\left(\nabla^{L C ; M}\right)_{\frac{\partial}{\partial x}} \overrightarrow{T_{[\gamma]} S}, v\right), w\right)_{\iota(s)}+g\left(J \nabla_{X}^{\perp} Y, w\right)_{\iota(s)} \\
=\varphi_{\chi}\left(\gamma^{*}\left(\nabla^{L C ; M}\right)_{\frac{\partial}{\partial x}} \overrightarrow{T_{[\gamma]} S}, v, w\right)_{\iota(s)},
\end{gathered}
$$

where we used $\nabla_{X}^{\perp} Y=0$ from Lemma 2.8.

Let $E_{1}(x, s), \cdots, E_{r}(x, s)$ be the basis of $T_{\gamma(x, s)} \gamma(x) S$ obtained by the exponential flow in the direction $X$ from an orthonormal basis $e_{1}(s), \cdots, e_{r}(s)$ of $T_{\iota(s)} \iota(S) \subset T_{\iota(s)} M$, so that

$$
\overrightarrow{T_{\gamma(x, s)} \gamma(x)(S)}=\operatorname{vol}\left(E_{1}(x, s) \wedge \cdots \wedge E_{r}(x, s)\right)^{-1} E_{1}(x, s) \wedge \cdots E_{r}(x, s) .
$$

Then we have

$$
\begin{aligned}
& \left.\varphi_{\chi}\left(\gamma^{*}\left(\nabla^{L C ; M}\right)_{\frac{\partial}{\partial x}} \overrightarrow{T_{[\gamma]} S}, v, w\right)\right|_{x=0} \\
& \quad=\gamma^{*}\left(\nabla^{L C ; M}\right)_{\frac{\partial}{\partial x}}\left(\left.\left(\operatorname{vol}\left(E_{1}(x, s) \wedge \cdots \wedge E_{r}(x, s)\right)^{-1}\right)\right|_{x=0} \varphi_{\chi}\left(e_{1}, \ldots e_{r}, v, w\right)\right. \\
& \quad+\sum_{i=1}^{r} \varphi\left(e_{1}, \cdots,\left.\gamma^{*}\left(\nabla^{L C ; M}\right)_{\frac{\partial}{\partial x}} E_{i}\right|_{x=0}, \cdots, e_{r}, v, w\right) .
\end{aligned}
$$

From the defining equation (2.23) for the gradient vector field $W$ we have

$$
\begin{aligned}
\gamma^{*}\left(\nabla^{L C ; M}\right)_{\frac{\partial}{\partial x}} & \left(\left.\left(\operatorname{vol}\left(E_{1}(x, s) \wedge \cdots \wedge E_{r}(x, s)\right)^{-1}\right)\right|_{x=0}\right. \\
& =-g\left(u, W_{[\iota]}\right),
\end{aligned}
$$

so equation (3.6) becomes

$$
\begin{aligned}
\varphi_{\chi} & \left(\left.\gamma^{*}\left(\nabla^{L C ; M}\right)_{\frac{\partial}{\partial x}} \overrightarrow{T_{[\gamma]} S}\right|_{x=0}, v, w\right) \\
& =-g\left(u, W_{[\iota]}\right) \varphi_{\chi}\left(e_{1}, \ldots e_{r}, v, w\right) \\
& +\sum_{i=1}^{r} \varphi_{\chi}\left(e_{1}, \cdots,\left.\gamma^{*}\left(\nabla^{L C ; M}\right)_{\frac{\partial}{\partial x}} E_{i}\right|_{x=0}, \cdots, e_{r}, v, w\right)
\end{aligned}
$$

Denote by $\left.\left(\gamma^{*}\left(\nabla^{L C ; M}\right) \frac{\partial}{\partial x} E_{i}\right)\right|_{x=0} ^{T}$ the projection of $\left.\left(\gamma^{*}\left(\nabla^{L C ; M}\right) \frac{\partial}{\partial x} E_{i}\right)\right|_{x=0}$ to $T \iota(S)$, and by $\left.\left(\gamma^{*}\left(\nabla^{L C ; M}\right) \frac{\partial}{\partial x} E_{i}\right)\right|_{x=0} ^{\perp}$ the projection on the normal bundle. As the Levi-Civita connection $\nabla^{L C ; M}$ is torsionless and compatible with the metric $g$ on $M$, we have

$$
\left.\left(\gamma^{*}\left(\nabla^{L C ; M}\right)_{\frac{\partial}{\partial x}} E_{i}\right)\right|_{x=0} ^{T}=\left(\nabla_{e_{i}}^{L C ; M} u\right)^{T}=\sum_{i=1}^{r} g\left(\nabla_{e_{i}}^{L C ; M} u, e_{j}\right) e_{j} .
$$


Therefore

$$
\begin{array}{r}
\sum_{i=1}^{r} \varphi_{\chi}\left(e_{1}, \cdots,\left.\gamma^{*}\left(\nabla^{L C ; M}\right)_{\frac{\partial}{\partial x}} E_{i}\right|_{x=0} ^{T}, \cdots, e_{r}, v, w\right) \\
=\left(\sum_{i=1}^{r} g\left(\nabla_{e_{i}}^{L C ; M} u, e_{i}\right)\right) \varphi_{\chi}\left(e_{1}, \cdots, e_{r}, v, w\right) .
\end{array}
$$

As $u$ is a normal vector field on $\iota(S)$ while $e_{i}$ is a tangent vector field, we have that $g\left(u, e_{i}\right)$ is identically zero on $S$ and so by applying $\nabla_{e_{i}}^{L C ; M}$ we find

$$
g\left(u,\left(\nabla_{e_{i}}^{L C ; M} e_{i}\right)^{\perp}\right)=-g\left(\nabla_{e_{i}}^{L C ; M} u, e_{i}\right) .
$$

By Lemma 2.9 we therefore get

$$
g\left(u, W_{[\iota]}\right)=\sum_{i=1}^{r} g\left(\nabla_{e_{i}}^{L C ; M} u, e_{i}\right)
$$

Equation (3.7) then reduces to

$$
\begin{aligned}
\varphi_{\chi} & \left(\left.\gamma^{*}\left(\nabla^{L C ; M}\right)_{\frac{\partial}{\partial x}} \overrightarrow{T_{[\gamma]} S}\right|_{x=0}, v, w\right) \\
& =\sum_{i=1}^{r} \varphi_{\chi}\left(e_{1}, \cdots,\left(\nabla_{e_{i}}^{L C ; M} u\right)^{\perp}, \cdots, e_{r}, v, w\right)
\end{aligned}
$$

and so we have obtained the identity

$$
g\left(\nabla_{u}^{\perp}(J Y), w\right)_{\iota(s)}=\sum_{i=1}^{r} \varphi_{\chi}\left(e_{1}, \cdots,\left(\nabla_{e_{i}}^{L C ; M} u\right)^{\perp}, \cdots, e_{r}, v, w\right)_{s}
$$

for ay sections $u, v, w$ of $N_{\iota} S$ and for any extension $Y$ of $v$ to a vertical vector field along a three-parameter deformation $\gamma$ of $\iota$ by equation (2.18). As for fixed $v$ and $w$ the left hand side of equation (3.9) only depends on the value of $u$ at $s$, it is not restrictive to assume $\left(\nabla_{e_{i}}^{L C ; M} u\right)_{s}^{\perp}=0$ for every $i=1, \ldots, r$. Therefore $g\left(\nabla_{u}^{\perp}(J Y), w\right)_{\iota(s)}=0$, as we wanted to prove.

Let us recall the expression of the Nijenhuis tensor $N_{J}$ of an almost complex structure $J$ on a smooth manifold $\mathfrak{M}$, see e.g., [KN1969, p. 123].

$$
N_{J}(X, Y):=2\{[J X, J Y]-[X, Y]-J[X, J Y]-J[J X, Y]\}
$$

for $X, Y \in \mathfrak{X}(\mathfrak{M})$. Moreover, if $\nabla$ is an almost complex affine connection with respect to the almost complex structure $J$, by Proposition 3.6 in [KN1969, p. 145], we have

$$
N_{J}(X, Y)=-2(T(J X, J Y)-J(T(J X, Y))-J(T(X, J Y))-T(X, Y)),
$$

where $T$ is the torsion of $\nabla$. It is noteworthy that the proof of Proposition 3.6 ibid. works not only for the case of finite dimensional manifolds $\mathfrak{M}$, but also for infinite dimensional manifolds for which affine connections and Lie brackets of vector fields can be defined. Namely, the argument ibid. uses the expression for $T$ defined in (2.8), and then applies it to the RHS 
of (3.11). Summing up, the RHS of (3.11) is written as a sum of eight summands involving $\nabla$ and four summands involving the Lie brackets. Since $\nabla$ commute with $J$ by definition of almost complex affine connection, the sum of the eight summands involving $\nabla$ vanishes. Then they observe that the sum of the four summands involving the Lie brackets is equal to the RHS of (3.10). This proves (3.11).

In particular, this applies to the infinite dimensional manifold $B_{i, f}^{+}(S, M)$ As $\nabla^{\perp}$ is torsion-free by Proposition 2.7, and it is almost complex with respect to the almost complex structure $J$ by Proposition 3.3 , we get the following.

Corollary 3.4. The almost complex structure $J$ on $B_{i, f}^{+}(S, M)$ satisfies $N_{J}=0$ and so it is a formally integrable almost complex structure.

Proposition 3.5. The almost complex structure $J$ is parallel with respect to the Levi-Civita connection $\nabla^{L C}$. Equivalently, $\nabla^{L C}$ is an almost complex connection with respect to the almost complex structure $J$.

Proof. We already know that $B_{i, f}^{+}(S, M)$ is a smooth manifold endowed with the formally integrable almost complex structure $J$, defined by (3.2), and with the $L_{2}$-metric, which is a compatible with $J$ an so defines a formally Kähler structure. As the fundamental 2-form $\omega$ of the formally Kähler metric is closed, by [KN1969, Proposition 4.2, p. 148] we then have

$$
4\left\langle\left(\nabla_{X}^{L C} J\right) Y, Z\right\rangle=\left\langle N_{J}(Y, Z), J X\right\rangle=0
$$

for any three vector fields $X, Y, Z$ on $B_{i, f}^{+}(S, M)$, hence $\nabla^{L C} J=0$.

Proof of Theorem 1.3. Theorem 1.3 follows from Proposition 3.5 and Corollary 3.4 .

\section{ACKNOWLEDGEMENT}

DF thanks the Institute of Mathematics of the Czech Academy of Sciences for hospitality and for the excellent research environment in which this article was started. DF and HVL thank Patrick Iglesias-Zemmour and Ioannis Chrysikos for stimulating and helpful discussions at the beginning of this project. We are grateful to the anonymous referees for critical helpful comments.

\section{REFERENCES}

[BBM2013] M. Bauer, M. Bruveris And P. W. Michor, Overview of the Geometries of Shape Spaces and Diffeomorphism Groups, Journal of Mathematical Imaging and Vision, 50(2014), 1-2, 60-97.

\footnotetext{
${ }^{1}$ Kobayshi-Nomidzu gave a proof of Proposition 4.2 ibid. that is also valid for infinite dimensional manifolds locally modeled on convenient vector spaces, since the standard formula for $d \omega$ ibid. is also valid in the convenient setting [KM1997, (3), p. 342].
} 
[BG1967] R.B. Brown, A. Gray, Vector cross products, Comment. Math. Helv. 42 (1967) 222-236.

[Brylinsky2008] J.-L. Brylinski, Loop Spaces, Characteristic Classes and Geometric Quantization, Birkhäuse, 2008, reprint of the 1993 Edition.

[CGN2018] D. Crowley, S. Goette And J. Nordstroem, Distinguishing $G_{2^{-}}$ manifolds, arXiv:1808.05585.

[CMm1991] V. Cervera, F. Mascaro, and P. W. Michor, The action of the diffeomorphism group on the space of immersions. Diff. Geom. Appl. , 391-401, 1991.

[Gray1969] A. GraY, Vector cross products on manifolds, Trans. Amer. Math. Soc. 141 (1969) 465-504.

[Henrich2009] F.-F. HenRICH, Loop spaces on Riemannian manifolds, PhD Thesis TU Berlin, 2009.

[Joyce2000] D. D. Joyce, Compact manifolds with Special Holonomy, Oxford University Press, 2000.

[Joyce2007] D. D. Joyce, Riemannian holonomy groups and calibrated geometry, Oxford Univ. Press, 2007.

[Kainz1984] G. Kainz, A Metric on the Manifold of Immersions and Its Riemannian Curvature, Mh. Math. 98, 211-217, (1984).

[KN1969] S. Kobayashi And K. Nomidzu, Foundations of differential Geometry, vol. 2, Intersciences Publishers, 1969.

[KM1997] A. Kriegl And P. W. Michor, The Convenient Setting of Global Analysis, AMS, 1997.

[LeBrun1984] C. LeBrun, Twistor CR manifolds and three-dimensional conformal geometry, Trans. Amer.Math. Soc 284 (1984) 601-616.

[LeBrun1993] C. LeBrun, A Kähler structure on the space of string worldsheets, Classical Quantum Gravity 10 (1993), no. 9, L141-L148.

[Lempert1993] L. LEMPERT, Loop spaces as complex manifolds, J. Differential Geom. 38 (1993), no. 3, 519-543.

[LL2007] J. H. Lee And N. C. Leung, Higher dimensional knot spaces for manifolds with vector cross products, Advances in Mathematics 213(2007), 140-164.

[Michor2016] P. Michor, Manifolds of mappings and shapes, In: The legacy of Bernhard Riemann after one hundred and fifty years. Editors: Lizhen Ji, Frans Oort, ShingTung Yau. ALM 35 (2016), 459-486. International Press Boston, arXiv:1505.02359.

[MM2005] P. W. Michor And D. Mumford, Vanishing geodesic distance on spaces of submanifolds and diffeomorphisms. Documenta Math. , 10:217-245, 2005.

[MMM2013] M. Micheli, P. Michor, D. Mumford, Sobolev metrics on diffeomorphism groups and the derived geometry of spaces of submanifolds. Izv. Ross. Akad. Nauk Ser. Mat. 77 (2013), no. 3, 109-138

[Verbitsky2010] M. Verbitsky, A formally Kähler structure on a knot space of a $G_{2}$ manifold, Sel. Math. New. Ser. (2012) 18:539-555, arXiv:1003.3174.

[Vizman2011] C. Vizman, Induced differential forms on manifolds of functions, Arch. Math. (Brno) 47 (2011), no. 3, 201-215.

Dipartimento di Matematica "Guido Castelnuovo", Università di Roma "LA Sapienza", Piazzale Aldo Moro 2, 00185 Roma, Italy

Email address: fiorenza@mat.uniroma1.it

Institute of Mathematics of the Czech Academy of Sciences, Zitna 25, 11567

Praha 1, Czech Republic

Email address: hvle@math.cas.cz 